\section{OPTO-MECHANICAL SYSTEM FOR LASER BEAM DISTRIBUTION} KAROLINA MACUCHOVA, JAN HERMANEK, MARTINA REHAKOVA

HiLASE Centre, Institute of Physics CAS, Dolni Brezany, Czech Republic

DOI : 10.17973/MMSJ.2019_12_2019106

karolina.macuchova@hilase.cz

Laser beam distribution requires complex opto-mechanical system allowing safe and precise delivery of different laser beams from hall laboratories to experimental workstations developed within the HiLASE project of the IOP in the Czech Republic. The unique nature of new generation of HiLASE high energy diode-pumped solid state laser systems with high repetition rates requires advanced design approach. We use modular approach that allows gradual modification and flexible change of the distribution layout according to current laboratory needs as well as customization of individual branches including their optical, mechanical and electronic equipment. The distribution system performance excels with high pointing stability and low vibration transmission.

KEYWORDS

Laser facility, laser beam distribution, high-power laser, optomechanical design, vacuum chamber, HiLASE

\section{INTRODUCTION}

The HiLASE center research focuses on development of various high-power laser systems for scientific and industrial applications. There are already more than twenty laser systems varying in wavelengths, pulse lengths, or pulse energies [Divoky 2014], [Rostohar, 2015]. Their main features are summarized in the Table 1.

\begin{tabular}{|c|c|c|c|c|}
\hline Laser & $\begin{array}{c}\text { Wavelength } \\
\text { [nm] }\end{array}$ & $\begin{array}{c}\text { Pulse } \\
\text { energy }\end{array}$ & $\begin{array}{c}\text { Pulse } \\
\text { width }\end{array}$ & $\begin{array}{c}\text { Repetition } \\
\text { rate [Hz] }\end{array}$ \\
\hline $\begin{array}{c}\text { PERLA } \\
\text { A }\end{array}$ & 1030 & $100 \mathrm{~mJ}$ & $<5 \mathrm{ps}$ & 100 \\
\hline $\begin{array}{c}\text { PERLA } \\
\text { B }\end{array}$ & 1030 & $\begin{array}{c}10-14 \\
\mathrm{~mJ}\end{array}$ & $\begin{array}{c}<1.6 \\
\mathrm{ps}\end{array}$ & 1000 \\
\hline BIVOJ & 1030 & $\begin{array}{c}10 / 100 \\
\mathrm{~J}\end{array}$ & $10 \mathrm{~ns}$ & 10 \\
\hline
\end{tabular}

Table 1. Parameters of the distributed lasers

The laser beam distribution system (LBDS) contains two independent beamlines. First beamline (L1) distributes the Perla A and Perla B thin-disk based lasers. Each beamline delivers laser pulses nolonger than few ps at different repetition rates and pulse energy. The second beamline (L2) distributes the high-energy output beam from the laser system called Bivoj.

These laser beams are delivered to experimental stations across the HiLASE facility. One of the experimental stations is called Laser Inducted Damage Threshold testing station (LIDT station) and is located on the ground floor. The other experimental station is Laser Shock Peening experimental station (LSP station) which is located in the first floor. The LSP station researches improvement of fatigue life, fretting fatigue and stress corrosion cracking of metal materials. While both the experimental stations run simultaneously, the LBDS layout was designed to allow parallel beam delivery (see Figure 1).

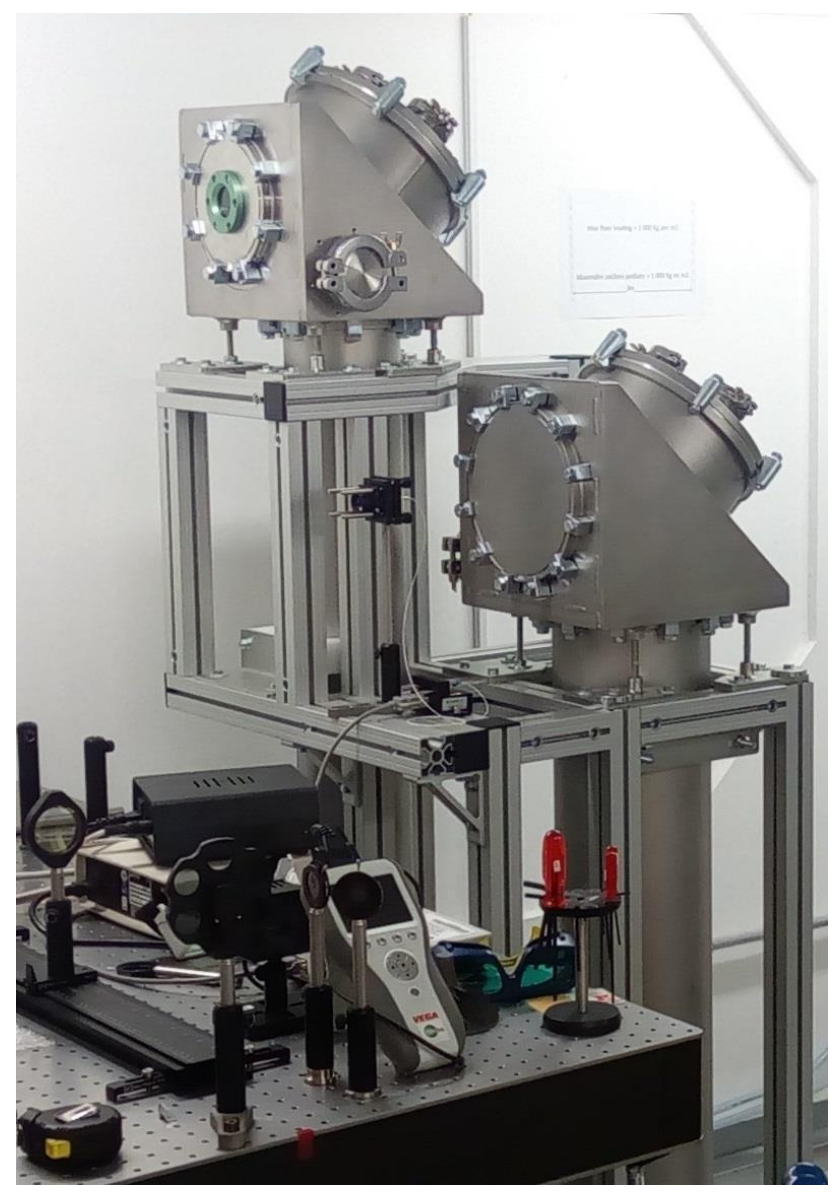

Figure 1. Parallel running beamlines, output of the LBDS in the LSP station

The LBDS can lead through ceiling penetrations in the laser hall. The inter-floor penetrations are designed to be hermetically closed, in order to serve as a bridge between cleanroom ISO class 7 laser laboratories and experimental halls with no cleanliness, temperature and humidity control. The whole laser beam distribution system installed in the HiLASE facility laboratories is displayed in Figure 2.

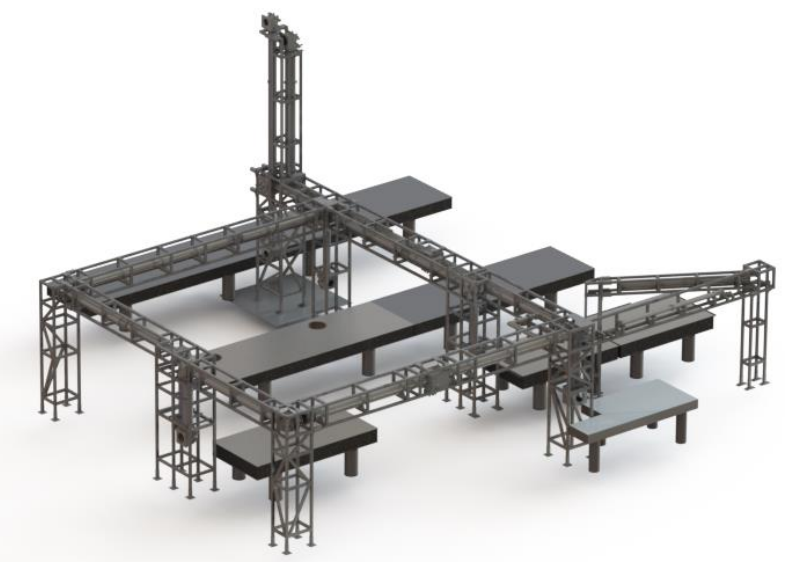

Figure 2. Overview of the whole LBDS installed within the HiLASE facility 


\section{OPTO-MECHANICAL SYSTEM}

The purpose of the distribution system is to allow an automated reconfiguration of the beam transport paths according to the requests for laser access for the experimental stations. At the same time, the LBDS has to preserve the wave quality and limit the diffraction limit, which could create large intensity peaks in the intensity profile and thus damage the optical coating. The relay imaging system has to keep the beam position fluctuation caused by pointing instability within the clear apertures.

\subsection{Folding mirror mounts}

Propagation of the laser beam to the target is via folding mirrors. For the Bivoj beamline, the beam is square-shaped with dimensions $25 \times 25 \mathrm{~mm}$ for $10 \mathrm{~J}$ pulse energy or $75 \times$ $75 \mathrm{~mm}$ for $100 \mathrm{~J}$ pulses, respectively. The Perla beamline transports a circular beam with $\varnothing 5 \mathrm{~mm}$ spot size.

The task of reflective transport mirrors is to change direction of the laser beam. Flat mirrors shape the optical path. They can also split the beam to allow diagnostics. The transport mirrors reflect the beam at $45^{\circ}$ angle of incidence. Size of the mirror is given by the beam size, power of the laser, damage threshold of the substrate and used coating layers. 6" mirrors are used for the $100 \mathrm{~J}$ Bivoj and 2" for $10 \mathrm{~J}$ Bivoj and Perla beamlines.

The reflective optics used in the system excels in high reflective surface which can be well suited for multispectral applications. High reflectivity for various wavelengths is reached by special metal or dielectric coating layers. Geometry of the surface is given by the application and cover up flat surface as well as aspheric.

The transport mirrors are mounted in holders on breadboard in vacuum chambers (see Figure 3 ).

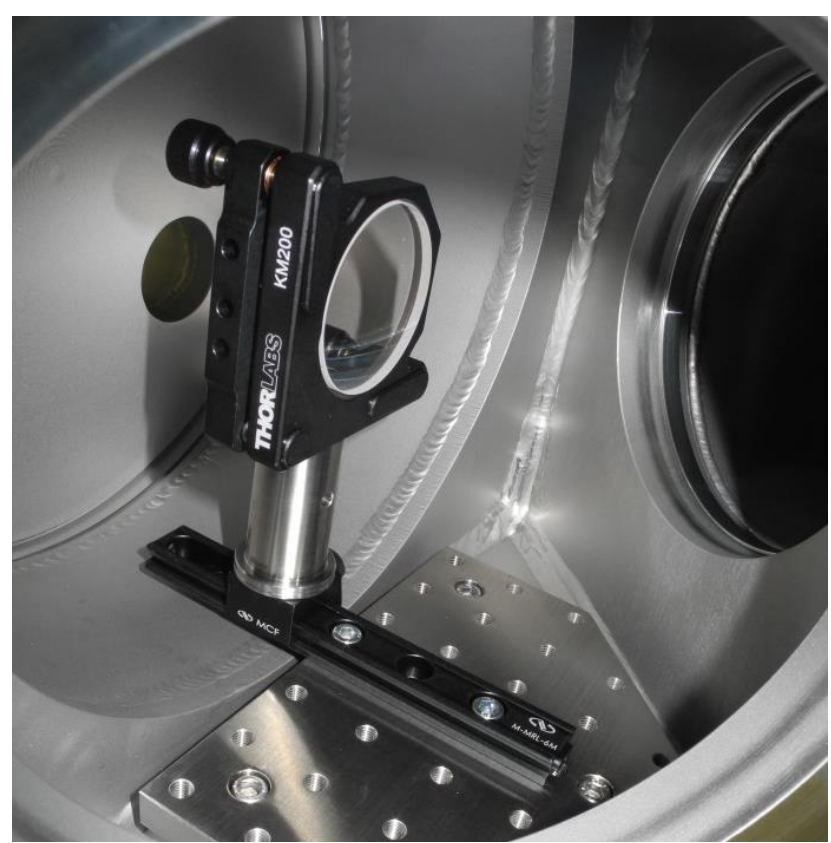

Figure 3. View of the inside of the triangular shaped chamber with a simple off-the-shelf opto-mechanical mirror holder

The opto-mechanical mount types in the system vary by degrees of freedom of adjustment. Each nod serves a slightly different purpose and thus has a different configuration. Main features which we should consider in the optical configuration are: field of view, performance and mounting requirements. [Fischer, 2008] Within calculation of the pointing error budget, we have considered following contributions: alignment accuracy, mounting error, thermal flexure, initial misalignment, and baseplate distortion.

We use principally two types of stainless steel vacuum chambers in the distribution system: triangular prism shaped and cuboid shape. The triangular prism shaped vacuum chambers are used for the stationary mirror reflectors. Each consists of a fold mirror with 3 degrees of freedom for alignment purposes. The alignment is done manually in air.

The larger cuboid shaped vacuum chambers are used to hold switching mirror mount that works as a fold one at $45^{\circ}$ and is combined with other adjustment mechanisms to allow pointing into different directions or a stage for moving the mount away from the beam path. These switchyard mirror mounts are automated with combination of a rotation or translation stage with incremental encoder and two fine actuators. In each beamline two mirror mount are equipped with a piezo driven actuation for precise beam pointing and stabilization.

Some of the opto-mechanical holders are off-the-shelf (see Figure 3) while the majority of them are made in-house (see Figure 4). For the Bivoj beamline we use 6" optical holders with reduction to $2^{\prime \prime}$ mirrors, so the beamline is easily switchable from $10 \mathrm{~J}$ distribution to $100 \mathrm{~J}$ and vice versa. Such an opto-mechanical mount is displayed in Figure 4.

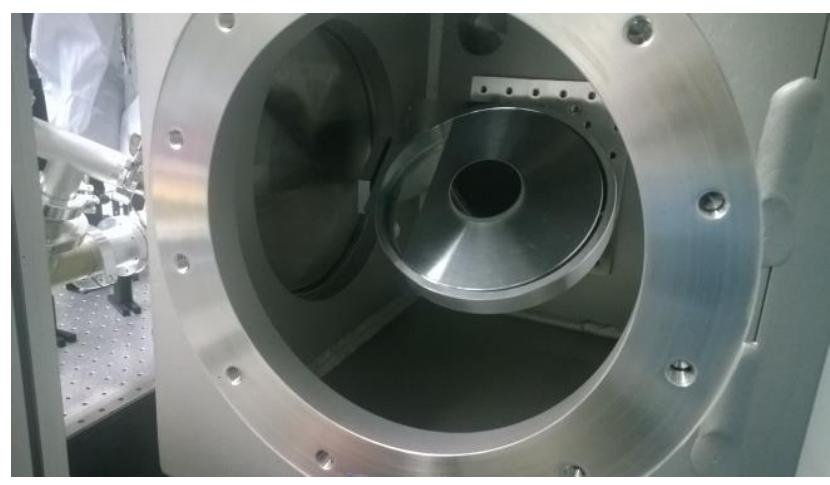

Figure 4. View of the inside of the cuboid chamber with a custom 6" mirror mount with reduction piece to $2^{\prime \prime}$

The alignment diagnostics is done using fiducials and flippers. Each vacuum chamber is equipped with a feedthrough for a diagnostic camera.

\subsection{Beam Imaging system}

Relay imaging system is involved to transport laser beam over long distances. The imaging system works as spatial filter. Spatial filter is an optical device that enables to filter noise and aberrations in the laser beam. These alterations of the beam profile occur due to imperfect, damaged or dusty optics, variations in the optical medium or dust in the air. The cleaned up laser beam contains only single transverse mode. A filter consists of lens and a pinhole. The spatial filter assembly is displayed in Figure 5.

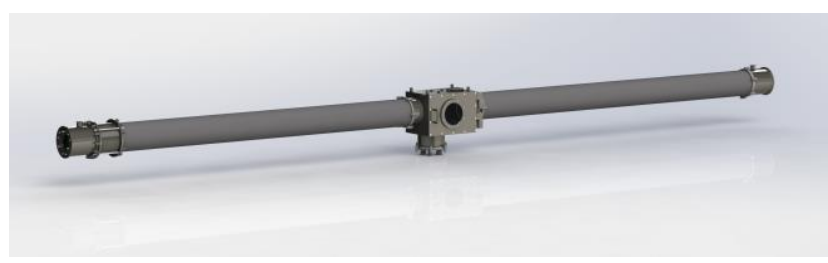

Figure 5. Imaging relay telescope 
The pinhole passes only fine central structure of the beam, while the distorted parts of the beam are filtered. [Yoder, 1992] The pinhole diameter is variable in range $\{2,3,4\} \mathrm{mm}$. From the mechanical point of view, the spatial filters are built on two fundamental parts: a pinhole chamber and two lens housings (one per each end) interconnected via tubes. The complete assembly of a vacuum spatial filter is displayed in Figure 6.

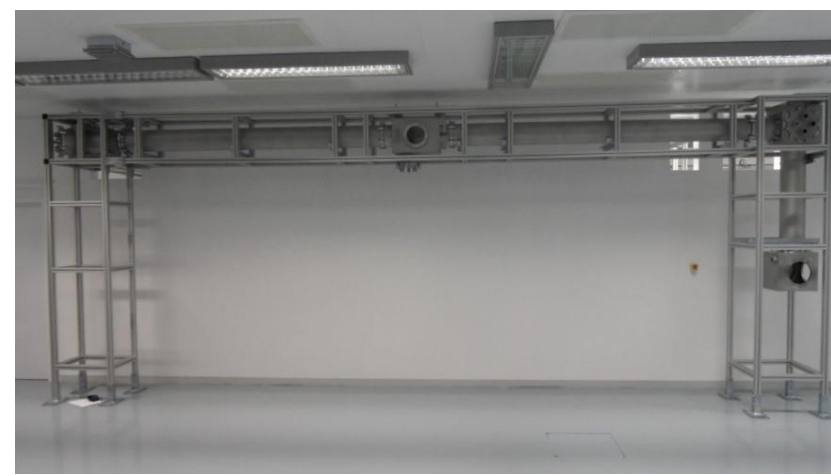

Figure 6. Assembled relay telescope in the laboratory

All components are made of stainless steel and are vacuum tight up to 0,01 $\mathrm{Pa}$ [Kohl, 1995] [Murray, 2000]. To preserve a highest vacuum level possible, the inner surface of a chamber is polished and every bolt is ventilated in order to prevent any possible air-containing gap.

A pinhole chamber (see Figure 7) is equipped with a breadboard used as a support for a pinhole positioning mechanism.

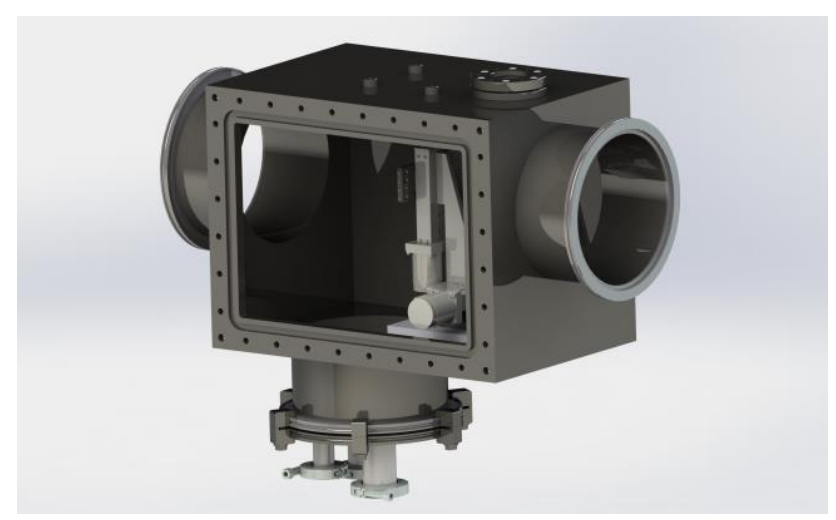

Figure 7. Pinhole chamber

The solution designed in HiLASE uses two-axis motorized setup in $X Y$ configuration for fine adjustment of the pinhole under vacuum. The adjustment in Z-axis (which is identical with the longitudinal axis of the spatial filter) does not require a high resolution movement and is therefore adjusted manually in air. The chamber also contains a periscopic mirror for a diagnostic camera output.

Lens housing design (see Figure 8 ) is based on two coaxial tubes with available positioning budget of $50 \mathrm{~mm}$ allowing to adjust lenses in the Z-axis direction for some minor corrections of the focal length of the telescope. Exact position of each lens is determined by a spacer and a setting flange.

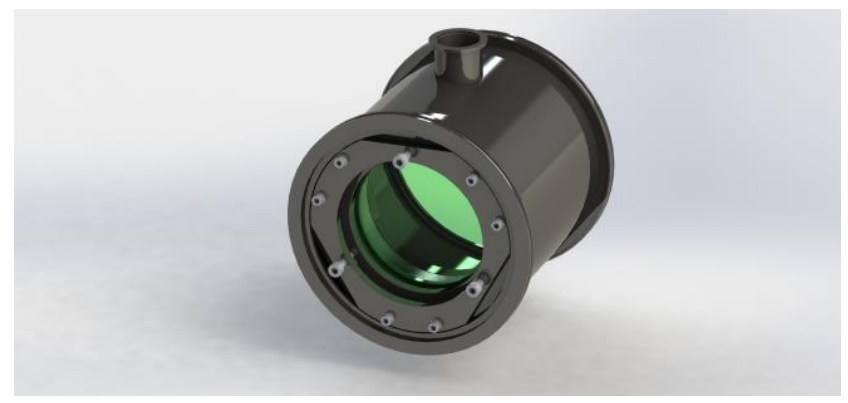

Figure 8. Tube lens holder

Lenses themselves are 6" plano-convex shaped with identical focal length for each spatial filter used in Bivoj beamlines. Therefore the imaging ratio is $1: 1$. The lenses also serve as a vacuum interface, eliminating the need of using another elements such as windows, which would spoil a beam profile.

The optical components used in the LBDS are very sensitive to the presence of dust particles, oil or other chemicals as these deteriorate the optical surface. Therefore we use dry vacuum pumps - a turbo pump by Pfeiffer (HiPace 300) for the spatial filters and a roughing pump (Pfeiffer ACP28) for the turbopump backing. A turbopump was selected for its low vibration and throttling while the multi-roots one was a frictionless design that provides reliability and low particulate exhaust in the clean-room environment. In addition, both pumps have a small footprint that in a clean room research laboratory can be an important factor due to increased maintenance costs.

Although a roughing pump with lower ultimate pressure could be used, the solution based on a turbo and roughing pump was selected due to the inherent pumping reserve and better flexibility. The turbopump can be throttled to achieve the desired vacuum level in the spatial filter with plenty of reserve in case of laser or distribution upgrades. This could also be used in case of a vacuum leak during an important experiment for a short-term pressure loss compensation. As the laser beam can be used in one experimental setup at a time, a single turbopump is enough for both vacuum filters. The pump is connected to the filters via a T piece, followed by gate valves. This setup allows selective pumping of the filters using just a single turbopump. The pressures in the VSF are monitored using two vacuum gauges - type Inficon MPG500.

\section{FRAMEWORK SUPPORT SYSTEM}

The opto-mechanical mirror holders are mounted through the vacuum chamber to a framework structure. The framework support system is assembled using the aluminium Kanya base extrusion type beams.

All support system framework is fixed only to the laser hall floor. The laser hall floor is designed as vibration free, so the transfer of vibrations from e.g. surrounding devices, water coolers, vacuum pumps, etc. is minimized. The whole LBDS including vacuum chambers, tubing, fixtures and framework support system is displayed in Figure 9. 


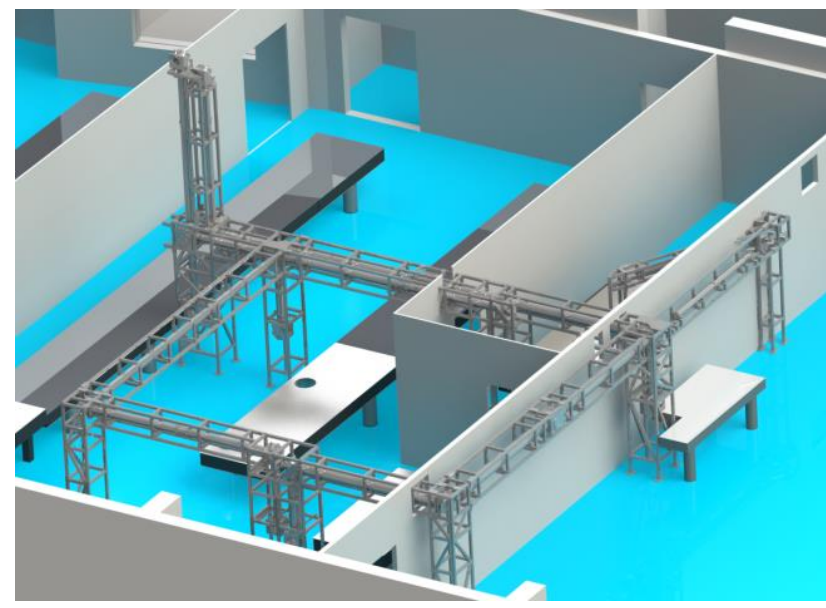

Figure 9. View on the LBDS in the laboratory

The framing is detached from any other part of the building. The framework support structure was calculated by computational software ANSYS using FEM (Finite Element Method) to ensure static load, dynamic performance and thermal stability. Evaluated structure was examined especially for thermal stability due to a different thermal expansion of aluminium support structure and stainless steel vacuum system. An installation of bellows that compensates larger thermal expansion of aluminium parts proved as very helpful.

\section{CONCLUSIONS}

A characteristic feature of the designed LBDS is already well proven modularity, which enables its gradual upgrades to accommodate specific needs of experimental projects as well as customization of individual branches including their optical, mechanical and electronic equipment. Its stability has been calculated using FEM and measured at both experimental stations. The results showed the LBDS to be very stable. For the $10 \mathrm{~Hz}, 1 \mathrm{~J}$ laser beam the transmitted energy fluctuation is so far the same as the laser output fluctuation.

The LBDS system was initially manufactured in 2015 by the local company Streicher, spol. $s$ r.o. and since then it was extended already two times: later in 2015 and 2019. The LBDS will undergo another major upgrade in the upcoming years to connect a new Bivoj beamline and deliver the laser beam to three new experimental stations.

\section{CONTACTS:}

Ing. Karolina Macuchová, Ph.D.

HiLASE Centre, Institute of Physics CAS

Za Radniciiiz28, 25241 Dolni Brezany, Czech Republic

+420-731-588-713, karolina.macuchova@hilase.cz, www.hilase.cz
Central vacuum system, personal safety system, automated control system, beam stabilization and diagnostics are being implemented and upgraded to a higher level of automation.

\section{ACKNOWLEDGMENTS}

This article was co-financed by the European Regional Development Fund and the state budget of the Czech Republic (project HiLASE CoE: Grant No CZ.02.1.01/0.0/0.0/15_006/0000674) and by the European Union's Horizon 2020 research and innovation programme under grant agreement No. 739573. This work was also supported by the Ministry of Education, Youth and Sports of the Czech Republic (Programmes NPU I Project No. LO1602, and Large Research Infrastructure Project No. LM2015086).

3D models were created in SOLIDWORKS under the University Research Edition license.

\section{REFERENCES}

[Divoky 2014] Divoky, M., Smrz, M., et al. Overview of the HiLASE project: High average power pulsed DPSSL systems for research and industry. In: High Power Laser Science and Engineering 2, 2014, doi:10.1017/hpl.2014.16.

[Fischer, 2008] Fischer, R. et al Optical System Design. USA: SPIE Press, McGraw-Hill Companies, Inc., 2008.

[Kohl, 1995] Kohl, W. Handbook of Materials and Techniques for Vacuum Devices. Woodbury: American Institute of Physics, 1995.

[Murray, 2000] Murray, J.E., Milam, D., et al, Spatial filter pinhole development for the National Ignition Facility, In: Appl. Opt. 39, 1405-1420, 2000.

[Rostohar, 2015] Rostohar, D., Lucianetti, A., et al HiLASE Project: high intensity lasers for industrial and scientific applications. In: P. Tománek, ed. Proc. SPIE 9450, Photonics, Devices, and Systems VI, 945002, January 6, 2015, http://dx.doi.org/10.1117/12.2070447.

[Yoder, 1992] Yoder, P. R. Opto-mechanical systems design. New York: Marcel Dekter, 1992. 